\title{
Termoterapia transpupilar em melanoma maligno da coróide
}

\author{
Transpupillary thermotherapy for malignant choroidal melanoma
}

\author{
Martha M. Motomo Chojniak ${ }^{1}$ \\ Tércio Guia ${ }^{2}$ \\ Fausto Uno ${ }^{3}$ \\ Clélia Maria Erwenne ${ }^{4}$
}

Trabalho realizado no Serviço de Oftalmologia do Hospital A. C. Camargo e Setor de Retina do Departamento de Oftalmologia da Universidade Federal de São Paulo (UNIFESP/EPM).

${ }^{1}$ Mestre e colaboradora do Setor de Ultra-som do Departamento de Oftalmologia da Universidade Federal de São Paulo; médica titular do Hospital A. C. Camargo.

${ }^{2}$ Tecnólogo do Setor de Retina - Departamento de Oftalmologia da Universidade Federal de São Paulo.

3 Doutor e Chefe do Setor de Retina do Departamento de Oftalmologia da Universidade Federal de São Paulo.

${ }^{4}$ Doutor e Chefe do Setor de Tumores - Hospital A. C. Camargo e da Universidade Federal de São Paulo.

Endereço para correspondência: Martha M. Motomo Chojniak - Alameda dos Aicás, 491/51 - São Paulo (SP) CEP 04086-001. E-mail: chojniak@uol.com.br

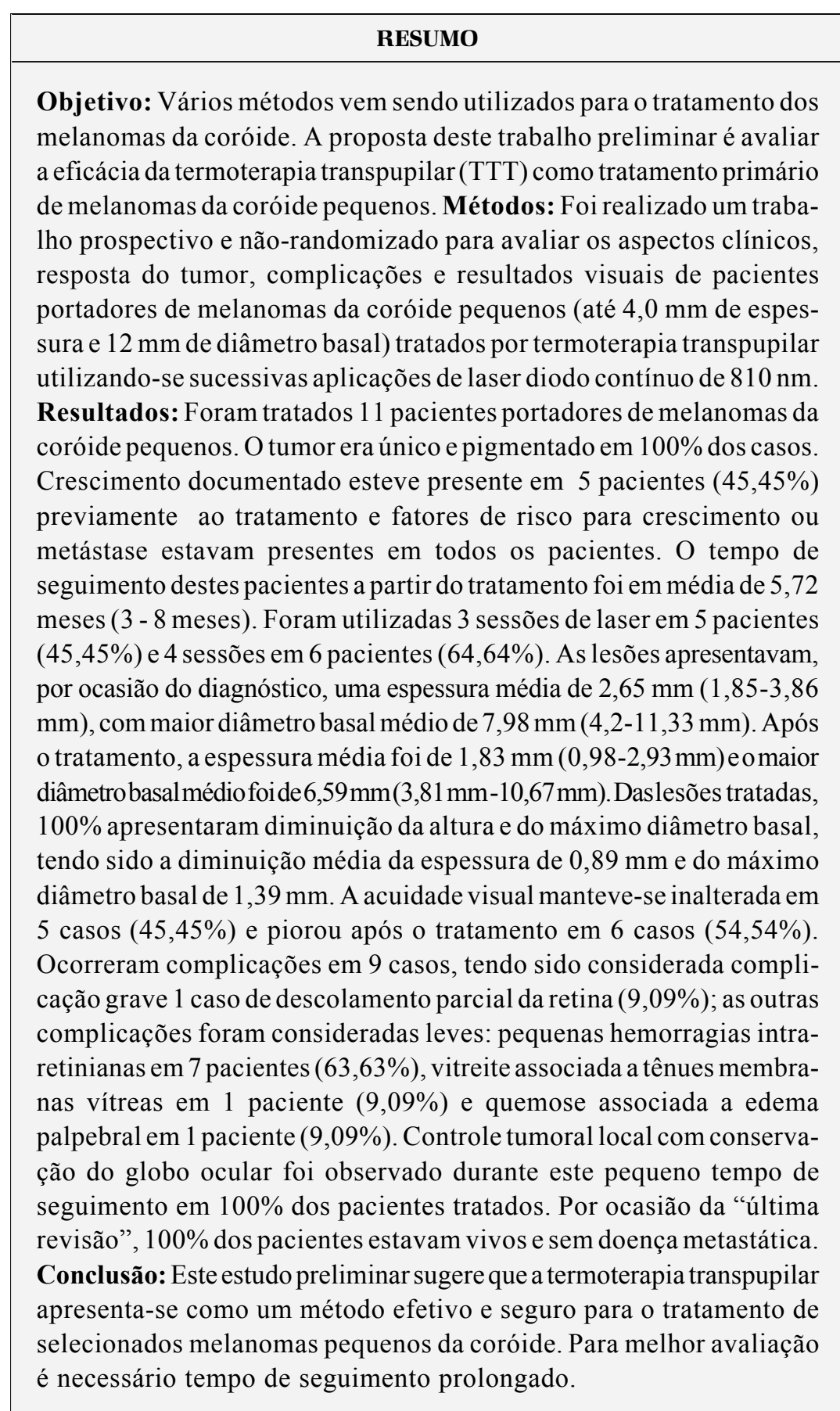

Descritores: Melanoma/terapia; Neoplasias da coróide/terapia; Hipertermia induzida/ métodos; Cirurgia a laser 


\section{INTRODUÇ̃̃OO}

O melanoma da úvea é o tumor intra-ocular primário mais freqüente, com origem nos melanócitos uveais derivados da crista neural. A incidência estimada é de seis novos casos/ milhão/ano. Acomete preferencialmente indivíduos acima dos 50 anos de idade e da raça branca, sendo raro nas raças negra e oriental. É essencialmente unifocal e unilateral. Pode acometer qualquer porção da úvea, sendo o da coróide posterior o mais comum; exibe grande variedade de padrões de crescimento e pigmentação, estando os sintomas e sinais clínicos na dependência do tamanho e localização da lesão, assim como de seu efeito às estruturas oculares adjacentes. $\mathrm{O}$ nevos, a melanocitose ocular congênita, a síndrome do nevos displásico e a neurofibromatose são considerados lesões predisponentes ao melanoma da úvea; outros fatores de risco questionados são: gravidez e cor clara da íris ${ }^{(1)}$.

O melanoma da úvea é um tumor agressivo, $30 \%$ dos pacientes vão a óbito devido à presença de doença metastática que ocorrem na maioria, dois a cinco anos após o diagnóstico e/ou tratamento. A via de disseminação é a hematogênica, sendo o fígado o órgão mais freqüentemente acometido, seguido pelo pulmão(1).

O melhor método terapêutico para o tratamento do melanoma da úvea ainda não está estabelecido. O principal fator determinante do tipo de tratamento a ser utilizado é o tamanho da lesão tumoral. Dentre as diferentes opções terapêuticas temos: observação periódica, fotocoagulação, hipertermia, radioterapia, incluindo a braquiterapia ou radioterapia por feixe externo de partículas carregadas, ressecção cirúrgica localizada, enucleação e exenteração ${ }^{(2)}$.

O manuseio dos pequenos tumores melanocíticos da coróide é assunto controverso. Uma importante razão para esta controversa é o fato de que o seu curso natural e potencial metastático não estão claramente definido. Baseado neste fato, muitos ainda acreditam que a melhor forma de manusear estes tumores pequenos seria a observação periódica até que crescimento documentado estivesse presente porém, alguns estudos vem demonstrando o potencial maligno destas lesões. Uma recente meta-análise mostrou que tumores classificados como melanomas pequenos apresentaram uma taxa de mortalidade de $16 \%$ em 5 anos $^{(3)}$.

Atualmente são bem reconhecidos alguns fatores de risco para crescimento e desenvolvimento de doença metastática em pequenas lesões melanocíticas da coróide, sendo sua presença indicativa de tratamento, são eles: crescimento documentado, localização de 0 a $3,0 \mathrm{~mm}$ a partir do nervo óptico, presença de líquido subretiniano, presença de pigmentos alaranjados na superfície do tumor e sintomas. Se 4 fatores estiverem presentes, o risco de metástase estimado é de $25 \%{ }^{(3)}$.

A termoterapia transpupilar (TTT) é um método recente que tem se revelado como uma eficaz forma de tratamento para os melanomas da coróide posterior considerados pequenos; o método utiliza radiação infravermelha como fonte de calor provocando a hipertermia do tecido tumoral, que por ter um sistema vascular irregular impede a boa disseminação do calor, aquecendo mais que os tecidos peritumorais.

Nuijs-Beems e col. ${ }^{(4)}$ e Jornee-de-Korvwer e col. ${ }^{(5-7)}$ foram os primeiros a relatar a utilização da hipertermia como tratamento primário e único em animais. Tanto Oostherhuis e col. ${ }^{\left({ }^{8}\right)}$ como Shields e col. ${ }^{(9-10)}$ obtiveram sucesso no tratamento de melanomas pequenos utilizando este método como forma única de tratamento em respectivamente 3,17 e 100 pacientes.

A termoterapia é utilizada para o tratamento de melanomas com até $4,0 \mathrm{~mm}$ de espessura. Para tumores maiores podemos associá-la à braquiterapia, geralmente sendo utilizada a $1^{\text {a }}$ sessão no pós-operatório imediato à retirada da placa radioterápica ${ }^{(5-7)}$.

\section{OBJETIVO}

Este trabalho prospectivo e não randomizado objetivou uma análise preliminar da resposta tumoral de uma amostra brasileira de portadores de melanoma maligno da úvea considerados pequenos tratados por termoterapia transpupilar utilizando-se laser diodo contínuo de $810 \mathrm{~nm}$, seus efeitos colaterais e os resultados visuais.

\section{MÉTODOS}

A nossa amostra foi constituída por um total de 11 pacientes portadores de melanomas da úvea pequenos (até 4,00 $\mathrm{mm}$ de espessura e $12 \mathrm{~mm}$ de diâmetro basal máximo) tratados por termoterapia transpupilar a partir de março de 1998 no Hospital do Câncer - Fundação Antonio Prudente em conjunto ao Setor de Retina do Departamento de Oftalmologia da Universidade Federal de São Paulo - Escola Paulista de Medicina (UNIFESP-EPM). Todos os pacientes incluídos nesta amostra apresentavam ou crescimento documentado da lesão ou fatores de risco para crescimento e/ou metástase ${ }^{(3)}$. Foram tratados primariamente e exclusivamente por termoterapia transpupilar.

Foram considerados como fatores de exclusão: tratamentos prévios; invasão tumoral da retina, disco óptico, vítreo ou esclera; hemorragia subretiniana ou opacidade de meios que impossibilitassem adequada observação do tumor e presença de doença metastática.

Os pacientes foram submetidos à avaliação oftalmológica e sistêmica completa que incluiu:

Avaliação oftalmológica: Medida da acuidade visual, medida da pressão intra-ocular, biomicroscopia, mapeamento da retina, ultra-sonografia ocular e angiografia fluoresceínica.

Avaliação sistêmica: Exame de sangue com dosagem de enzimas hepáticas (DHL, TGO, TGP, Gama GTP, Fosfatase Alcalina, Bilirrubinas Totais, Direta e Indireta), ultra-som de abdome superior e Raios-X de tórax PA e Perfil.

A avaliação oftalmológica foi realizada previamente ao tratamento, antes de cada nova sessão da TTT, no final do 
tratamento e trimestralmente a partir desta data, excetuandose o estudo angiofluoresceinográfico que foi realizado somente antes do tratamento e no final do mesmo. A avaliação sistêmica foi realizada previamente ao tratamento e semestralmente a partir desta data.

Os dados colhidos em relação à amostra foram: idade, raça, sexo, acuidade visual, pressão intra-ocular, tamanho do tumor (espessura e diâmetros basais antero-posterior e latero-lateral obtidos em milímetros- Ultra-som de $10 \mathrm{MHz}$ da Humphrey), localização meridional do centro do tumor (nasal, superior, temporal, inferior ou macular), pigmentação (amelanótico, levemente pigmentado ou densamente pigmentado); fluido subretiniano (presente ou ausente); pigmentos alaranjados na superfície do tumor (presente ou ausente); drusas (presente ou ausente); sintomatologia (presente ou ausente); crescimento documentado(presente ou ausente).

O tratamento era constituído por sessões de laser diodo infravermelho contínuo de $810 \mathrm{~nm}$ (IRIS Medical), com mira de $3,0 \mathrm{~mm}$, potência de $500 \mathrm{~mW}$ e tempo de exposição de 1 minuto por ponto, aplicados por toda a superfície do tumor de forma sobreposta e concêntrica, iniciando-se da periferia em direção ao centro da lesão, incluindo 1,0 a 2,0 mm de tecido coroidal livre junto à margem tumoral (margem de segurança). Foram realizadas pelo menos 3 sessões de TTT por paciente, com intervalo médio de 6 semanas.

A aplicação do laser era realizada sob anestesia local peribulbar utilizando-se $3 \mathrm{ml}$ de xilocaína a $\%$ sem vasoconstrictor $+3 \mathrm{ml}$ de marcaína a $0,5 \%$ sem vasoconstrictor $+1,0 \mathrm{ml}$ de hialozima, sendo injetado $4 \mathrm{ml}$ junto ao rebordo orbitário superior e $4 \mathrm{ml}$ junto ao inferior. Após cada sessão de laser o paciente permaneceu com curativo oclusivo por um período de 24 horas. Eram prescritos colírio midriático $\left(\right.$ Mydriacyl ${ }^{\circledR}$ ) a cada 8 horas e colírio antibiótico e antiinflamatório (Maxitrol ${ }^{\circledR}$ ) a cada 6 horas, ambos por um período de 7 dias. Para avaliação da eficácia do tratamento, foi considerada resposta favorável a obtenção clínica de uma cicatriz corioretiniana atrófica associada a diminuição, ou pelo menos não evolução do tamanho da lesão ao ultra-som e diminuição da vascularização intrínseca da lesão ao estudo angiofluoresceinográfico comparativo pré e pós-tratamento.

Após as 3 primeiras sessões de TTT era avaliada a resposta do tumor ao tratamento. Na ausência de favorável padrão de regressão clinicamente ou ao ultra-som, assim como na manutenção de vascularização intrínseca, adicionais sessões de TTT eram realizadas.

\section{RESULTADOS}

Foram tratados 11 pacientes portadores de melanomas da coróide pequenos por meio da termoterapia transpupilar utilizando laser diodo contínuo de $810 \mathrm{~nm}$. Destes, 5 (45,45\%) eram do sexo feminino e $6(54,54 \%)$ do sexo masculino. A média de idade apresentada por ocasião do diagnóstico foi de 58,90 anos (39-78 anos). A raça branca representou $100 \%$ da amostra. O olho direito foi acometido em 6 casos $(54,54 \%)$ e o esquerdo em $5(45,45 \%)$.

Sintomas estiveram presentes em todos os pacientes $(100 \%)$. Houve baixa da acuidade visual em 8 pacientes $(72,72 \%)$, moscas volantes em $2(18,18 \%)$ e fotopsias em 1 $(9,09 \%)$.

Crescimento documentado esteve presente em 5 pacientes $(45,45 \%)$ previamente ao tratamento.

A acuidade visual pré-tratamento variou de 0,8 a movimentos de mãos (MM) e pós-tratamento de 0,4 a movimentos de mãos (MM), tendo sido pior que 0,1 em 4 casos $(36,36 \%)$ por ocasião do diagnóstico, e em 6 casos $(54,54 \%)$ após o tratamento. A acuidade visual manteve-se a mesma em 5 casos $(45,45 \%)$ e piorou em 6 casos $(54,54 \%)$ após o tratamento.

A pressão intra-ocular variou de 10 a 14 mmHg pré-tratamento e de 8 a $13 \mathrm{mmHg}$ após o tratamento, não tendo sido detectado nenhum caso de glaucoma.

O tumor era único e pigmentado em 100\% dos casos. Pigmentos alaranjados na superfície do tumor estiveram presentes em 4 casos $(36,36 \%)$ e drusas estiveram presentes em 2 casos $(18,18 \%)$.

Em relação a localização das lesões, 5 (45,45\%) ocupavam o quadrante temporal, $3(27,27 \%)$ o quadrante temporal inferior, $2(18,18 \%)$ o quadrante temporal superior e $1(9,09 \%)$ o quadrante nasal inferior.

O pólo posterior estava acometido em 6 casos $(54,54 \%)$ e a região do equador em $5(45,45 \%)$, tendo sido acometida especificamente a região macular em 2 casos $(18,18 \%)$, perimacular em $2(18,18 \%)$ e peripapilar em $2(18,18 \%)$.

As lesões apresentavam, por ocasião do diagnóstico, uma espessura média de 2,65 mm (1,85-3,86 mm), com maior diâmetro basal médio de 7,98 mm (4,02-1,33 mm). Após o tratamento, a espessura média foi de $1,83 \mathrm{~mm}(0,98-2,93 \mathrm{~mm})$ e o maior diâmetro basal médio foi de 6,59 mm (3,81 mm - 10,67 mm). Das lesões tratadas, $100 \%$ apresentaram diminuição média da espessura de 0,89 mm e do máximo diâmetro basal de $1,39 \mathrm{~mm}$.

Ocorreram complicações em 8 casos $(72,72 \%)$. Foi considerada complicação grave a ocorrência em 1 paciente $(9,09 \%)$ de descolamento parcial da retina após o tratamento. As outras complicações foram consideradas leves: pequenas hemorragias intra-retinianas em 7 pacientes $(63,63 \%)$, vitreíte associada a tênues membranas vítreas em 1 paciente $(9,09 \%)$ e quemose associada a edema palpebral em 1 paciente $(9,09 \%)$.

Em 5 pacientes $(45,45 \%)$ foram realizadas 3 sessões de TTT e em 6 pacientes $(64,64 \%)$ foram necessárias 4 sessões, sendo que em 3 destes $(27,27 \%)$ a $4^{a}$ sessão foi realizada por não ter sido obtido o padrão de regressão tumoral considerado ideal pelo mapeamento de retina e ultra-sonografia e nos outros $3(27,27 \%)$, a mesma foi realizada após análise comparativa do estudo angiofluoresceinográfico pré e póstratamento, pois se detectou vascularização intralesional residual.

Por ocasião da "última revisão", 100\% dos pacientes estavam vivos e sem doença metastática. 
O tempo de seguimento a partir da data do tratamento foi em média de 5,72 meses (3 - 8 meses).

\section{DISCUSSÃO}

Durante muito tempo acreditou-se que a melhor forma de manipular os pequenos tumores melanocíticos da coróide a observação periódica, esperando até que sinais de malignidade como crescimento documentado estivessem presentes para que fosse instituído tratamento. Esta forma de raciocínio, entretanto é contrária à filosofia aplicada para os cânceres em geral que preconiza o diagnóstico e tratamento precoce com intuito de aumentar a sobrevida dos pacientes. O curso natural destas pequenas lesões melanocíticas da coróide vem sendo estudado e fatores preditivos de crescimento vêm sendo definidos, porém atualmente, mais do que a preocupação com o crescimento destas lesões, estudos vem sendo conduzidos com o objetivo de definir seu potencial de desenvolver doença metastática e morte $\mathrm{e}^{(11-16)}$.

Diener-West e col. encontraram uma taxa de mortalidade de $16 \%$ em 5 anos para portadores de tumores da coróide classificados como pequenos, e demonstraram que indivíduos com melanomas da coróide pequenos apresentam um risco de óbito 1,3 vezes maior quando comparados à população geral ${ }^{(13)}$.

Shields e col., analisando 1329 pacientes portadores de pequenos tumores melanocíticos da coróide (com menos de $3,0 \mathrm{~mm}$ de espessura), demonstraram que $18 \%$ deles apresentam crescimento e que 3\% desenvolvem doença metastática. Alguns fatores preditivos de crescimento e desenvolvimento de doença metastática para estes tumores foram definidos: maior espessura, margem posterior do tumor tocando o disco óptico, sintomas (flashes, moscas volantes, borramento da visão), pigmentos alaranjados na superfície da lesão e presença de fluido subretiniano.

Demonstraram que um tumor melanocítico pequeno da coróide com crescimento documentado apresenta chance de desenvolver metástase 8 vezes maior do que aquele que não apresenta crescimento, e que o risco de metástase multiplica quando mais de um fator de risco estiver presente, por exemplo, uma lesão de $1,0 \mathrm{~mm}$ com crescimento documentado tem risco de metástase 28 vezes maior que uma lesão com menos de $1,0 \mathrm{~mm}$ e sem evidencia de crescimento. Para entendermos o impacto de cada fator de risco temos que uma lesão com mais de $1,0 \mathrm{~mm}$ e que toca o disco óptico desenvolve metástase em $14 \%$ dos casos, se associado existir crescimento documentado o índice é de $17 \%$ e se associado tiver a presença de sintomas é de $25 \%$. Portanto, apesar de existir uma tendência à observação, aguardar por crescimento pode estar associado a um maior risco de metástase, sendo talvez a conduta mais razoável a instituição de tratamento preventivo em lesões de alto risco, com intuito de evitar transformação maligna e melhorar a sobrevida ${ }^{(3)}$.

Outra questão duvidosa é qual seria o tratamento mais adequado a ser utilizado.
A hipertermia vem sendo utilizada para o tratamento de lesões melanocíticas coroidais pequenas. Os sistemas que determinam hipertermia como forma de terapêutica, também chamados termoterapia, incluem: ultra-som, microondas, campos de corrente localizados, sementes térmicas ferromagnéticas e irradiação infravermelha (laser Diodo contínuo).

Diferente da fotocoagulação, onde a temperatura do tecido atinge níveis superiores à $65^{\circ} \mathrm{C}$, que causa necrose direta devido à coagulação, na TTT são utilizadas temperaturas subcoaguláveis, onde a necrose ocorre alguns dias após sua utilização devido à quebra mitocondrial ${ }^{(2)}$. São realizadas sucessivas aplicações de laser infravermelho em baixa potência e longa exposição, que submete o tumor a um regime de aquecimento (cozimento) que provoca a necrose celular do tumor por um aumento na temperatura do mesmo a níveis de $45^{\circ}$ a $60^{\circ} \mathrm{C}$. Quando utilizada em associação a braquiterapia, este método tem como objetivo aumentar a temperatura do melanoma para uma faixa de 42 a $44^{\circ} \mathrm{C}$, potencializando os efeitos da radioterapia e minimizando os efeitos da radiação ao tecido ocular normal.

A termoterapia transpupilar é, portanto uma técnica nova que utiliza radiação infravermelha como fonte de calor para tratar certos tumores oculares, dentre eles o retinoblastoma e o melanoma da coróide. A técnica utilizada para ambos é diferente, pois, sendo o retinoblastoma menos pigmentado, a TTT é utilizada em associação a quimioterapia, objetivando nestes casos proporcionar um distúrbio da integridade celular do tumor de forma a permitir a penetração do quimioterápico e prevenir o reparo do DNA, oferecendo uma taxa de controle tumoral acima de $90 \%$ para selecionados retinoblastomas. Nos casos de melanomas da coróide, o tratamento é mais agressivo, pois objetiva um efeito citotóxico direto, levando à necrose celular. Tem sido documentado que a penetração do calor atinge aproximadamente $4 \mathrm{~mm}$ dentro do tumor, induzindo necrose ao longo deste caminho de absorção ${ }^{(8)}$. Para os melanomas a técnica utilizada é a de se liberar calor por toda a superfície do tumor utilizando grandes miras de um tipo de laser de diodo modificado, de baixa potência e longa exposição $(810 \mathrm{~nm})$ sobrepostas, com duração de 1 minuto cada, perfazendo um tempo total médio de tratamento de 20 a 40 minutos. A TTT deve ser utilizada também em pelo menos 1,0 a $1,5 \mathrm{~mm}$ de tecido coroidal circundante de forma a criar-se uma margem de segurança evitando-se recorrências ou recidivas junto às margens tumorais.

A TTT apresenta uma regressão tumoral rápida quando comparada a braquiterapia de tumores com tamanho semelhante, ocorrendo a regressão para uma cicatriz corioretiniana plana em 3 a 6 meses.

O índice de controle tumoral observado em nossa amostra neste pequeno tempo de seguimento em portadores de melanomas pequenos tratados por termoterapia transpupilar foi de $100 \%$.

Shields ${ }^{(10)}$ relatou o resultado de 100 pacientes tratados, tendo encontrado um índice de regressão tumoral de 94\%. Situações que cursaram com regressão tumoral parcial ou 
ausente em sua amostra foram o tratamento de tumores amelanóticos e perifoveais, onde foi utilizado tratamento leve junto à margem foveolar com objetivo de preservação visual. Para a obtenção de controle tumoral nos tumores amelanóticos preconiza-se a associação da TTT ao corante indocianina verde com objetivo de aumentar a absorção do calor.

Algumas considerações sobre este tratamento é que os tumores pigmentados apresentam melhor resposta ao TTT quando comparada aos não-pigmentados. A presença de fluido subretiniano sobre a superfície do tumor menor que 3 $\mathrm{mm}$ não causa qualquer distúrbio no tratamento, porém se maior que $3 \mathrm{~mm}$, dispersão do calor com conseqüente menor efetividade pode ocorrer, além da possibilidade de se induzir uma atrofia retiniana pelo calor com subseqüente formação de buraco macular.

Em comparação a radioterapia, as complicações da TTT geralmente permanecem limitadas à área de tratamento. Obstrução vascular por resultado direto da esclerose vascular causada pelo calor pode ocorrer meses após o tratamento. Neovascularização da retina ocorre principalmente se existir obstrução da veia central da retina, podendo ser tratada por panfotocoagulação retiniana. Tração da retina desenvolve-se principalmente no tratamento de tumores de localização temporal e macular, além de ser mais freqüente naqueles tumores mais distantes do disco óptico.

$\mathrm{O}$ índice total de complicações encontrado em nossa amostra foi de $72,72 \%$, tendo sido considerada grave somente o desenvolvimento de descolamento parcial da retina de 1 paciente $(9,09 \%)$.

As principais vantagens do TTT em relação a braquiterapia são: redução tumoral mais rápida, tratamento mais focalizado com menor destruição do tecido normal, ausência de necessidade de hospitalização e anestesia geral e menor custo. Apesar dos resultados visuais até o momento serem animadores, com preservação ao redor de $50 \%$ na literatura e de $45,45 \%$ nesta amostra, com conservação do globo ocular em $100 \%$ dos casos, o tempo de seguimento dos pacientes é pequeno devendo ser lembrado que os efeitos visuais dos danos induzidos pelo calor podem se tornar clinicamente evidentes meses até anos após o tratamento, de forma similar à radioterapia. Dentre as desvantagens temos as múltiplas sessões necessárias, a imediata destruição da retina suprajacente, e a impossibilidade de se tratar tumores grandes ou de localização periférica.

O tratamento pela termoterapia transpupilar é feito em sessões. Shields acredita que a necrose tumoral máxima ocorra entre a $1^{\mathrm{a}}$ e a $2^{\mathrm{a}}$ sessão de laser, porém a $3^{\mathrm{a}}$ sessão é freqüentemente utilizada para maior segurança no controle tumoral. $\mathrm{O}$ resultado final é de uma cicatriz corioretiniana atrófica e plana ou escavada; freqüentemente observa-se presença de pigmentos planos e residuais que se acredita corresponder a tecido necrótico e que podem desaparecer ao longo do tempo. Em nossa amostra foram utilizadas 3 sessões de TTT em 5 pacientes $(45,45 \%)$ e em 6 pacientes $(64,64 \%)$ foram necessárias 4 sessões, para obtenção de padrão de regressão tumoral favorável.
Num pequeno tempo de seguimento, portanto, a TTT vem se apresentando como uma opção ao tratamento de melanomas pequenos (até 4,0 mm sem esclera), localizados principalmente junto ao pólo posterior (equador ou pós-equatorial). Um seguimento adequado de 10 a 15 anos será necessário para análise definitiva em relação à preservação visual, controle tumoral local e prognóstico sistêmico.

\section{ABSTRACT}

Purpose: Several methods have been used for treatment of choroidal melanoma. The purpose of this preliminary paper is to evaluate the effectiveness of transpupillary thermotherapy (TTT) as a primary treatment of small choroidal melanomas. Methods: This is a prospective nonrandomized study evaluating clinical aspects, tumor response, complications and visual outcome in patients presenting small choroidal melanomas (up to $4.0 \mathrm{~mm}$ thick and $12 \mathrm{~mm}$ base diameter) treated with TTT over $810 \mathrm{~nm}$ laser diode applications. Results: There were 11 patients treated with transpupillary thermotherapy, all of them presenting pig-mented small choroidal melanomas. Growth previous to treatment was documented in 5 patients and risk factors for growth or metastatic disease was present in all the patients. After treatment the patients were followed for 3 to 8 months (mean 5.7 months). Three laser sessions were used in 5 pa-tients and 4 sessions in 6 patients. The lesions presented at the beginning of the treatment a mean thickness of $2.7 \mathrm{~mm}$, with a mean larger base diameter of $7.8 \mathrm{~mm}$. All the lesions responded to treatment and presented decrease of thickness and base diameters. After transpupillary thermotherapy, the lesions' mean thickness was $1.8 \mathrm{~mm}$ and the mean larger base diameter was $6.7 \mathrm{~mm}$. The mean reduction in thickness was $0.9 \mathrm{~mm}$ and the mean decrease in larger base diameter was $1.4 \mathrm{~mm}$. The visual acuity remained unaffected in 5 cases and worsened after treatment in 6 cases. Nine patients presented compli- cations. A major complication occurred in a single patient who presented retinal detachment. Minor complications were observed as follows: small retinal hemorrhages (7 patients), vitreous inflammation associated with tenuous vitreous membranes ( 1 patient) and conjunctiva and eyelid edema (1 patient). Local tumor control and conservation of the eyeball was accomplished in all the patients; they are all alive without evidence of metastatic disease in this initial follow-up period. Conclusion: This preliminary study suggests that the transpupillary thermotherapy is a promising, effective and safe method for treatment of selected small choroidal melanomas. Further studies with longer follow-up period are necessary to better evaluate this treatment.

Keywords: Melanoma/therapy; Choroid neoplasms/therapy; Induced hyperthermia/methods; Laser surgery 


\section{REFERÊNCIAS}

1. Shields C, Shields JA. Introduction to melanocytic tumors of the uvea. In: Shields C, Shields JA. Intraocular tumors: a text and atlas. Philadelphia: Saunders. 1992. p. 45-59.

2. Shields C, Shields JA. Management of posterior uveal melanoma. In: Shields C, Shields JA. Intraocular tumors: a text and atlas. Philadelphia: Saunders. 1992. p. 171-205.

3. Shields C, Shields JA, Kiratli H, DePotter P, Cater JR. Risk factors for growth and metastasis of small choroidal melanocytic lesions. Ophthalmology 1995;102:9,1351-61.

4. Nuijs-Beems EM, Oosterhuis JA, Verburg-Van der Marel EH, WolffRouendaal D, Van Delft JL, Van Best JA. Tumor destruction by intermediate level hyperthermia. Curr Eye Res 1990;9:771-80.

5. Journee-de-Korvwer JG, Oosterhuis JA, Van Best J, Fakkel J. Xenon-arc phothocoagulator used for transpupillary thermotherapy. Doc Ophthalmol 1991;78:183-7

6. Journee-de-Korver JG, Oosterhuis JA, Kakebeeke-Kemme HM, WolffRouendaal D. Transpupillary thermotherapy (TTT) by infrared irradiation of choroidal melanoma. Doc Ophthalmol 1992;82:185-91.

7. Journee-de-Korver JG, Verburg van der Marrel EH, Oosterhuis JA et al. Tumoricidal effect of hiperthermia by near infrared irradiation on pigmented hamster melanoma. Lasers Light Ophthalmol 1992;4:175-80.
8. Oosterhuis JA, Journee-de-Korver JG, Kakebeeke-Kemme HM, Bleeker JC. Transpupillary thermotherapy in choroidal melanoma. Arch Ophthalmol 1995;113:315-21.

9. Shields CL, Shields JA, De Potter P, Kheterpal S. Transpupillary thermotherapy in the management of choroidal melanoma. Ophthalmology 1996; 103:1642-50

10. Shields CL, Shields JA, Caer J, Lois N, Edelstein C, Gunduz K, et al. Transpupillary thermotherapy for choroidal melanoma: tumor control and visual results in 100 consecutive cases. Ophthalmology 1998;105:581-90.

11. Gass JDM. Observation of suspected choroidal and ciliary body melanomas for evidence of growth prior to enucleation. Ophthalmology 1980;87:523-8.

12. McLean IW, Foster WD, Zimmerman LE, Martin DG. Inferred natural history of uveal melanoma. Invest Ophthalmol Vis Sci 1980;19:760-70.

13. Diener-West M, Hawkins BS, Markowitz JA, Schachat AP. A rewiew of mortality from choroidal melanoma II. A meta-analysis of 5-year mortality rate following enucleation, 1966 through 1988. Arch Ophthalmol 1992;110:245-50

14. Mims III JL, Shields JA. Follow-up studies of suspicious choroidal nevi. Ophthalmology 1978;85:929-43.

15. Augsburger JJ, Schroeder RP, Territo C, Gamel JW, Shields JA. Clinical parameters predictive of enlargment of melanocytic choroidal lesions. $\mathrm{Br} \mathrm{J}$ Opthalmol 1989;73:911-7.

16. Butler P, Char DH, Zarbin M, Kroll S. Natural history of indeterminate pigmented choroidal tumors. Ophthalmology 1994;101:710-7.

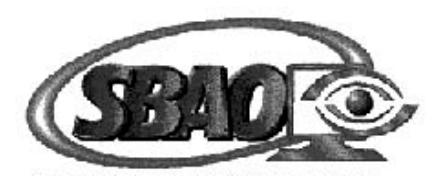

Sociedade Brasileira de Administração em Oftalmologia

\section{Congresso Brasileiro de Administração Profissional em Oftalmologia}

\author{
Promoção da ética e do exercício profissional
}

6 e 7 de julho de 2001 - São Paulo

\author{
SBAO: Alameda Santos, 1343 - Conj. $611-6^{\circ}$ Andar - Cerqueira César \\ Cep: 01419-001 - São Paulo - SP \\ Fone / Fax: (0xx11) 3266-4538 \\ JDE Eventos: Alameda Santos, $1343-$ Conj. $304-3^{\circ}$ andar - Cerqueira César \\ CEP: 01419-001 \\ Fone: (11) 287-9699 - 287-8109 - 289-4301 \\ Fax: (1 1) 288-8157 \\ E-mail: jdecomev@uol.com.br
}

